\title{
Non-linear Sorenson-Dice Exemplar Image Inpainting Based Bayes Probability for Occlusion Removal in Remote Traffic Control
}

\author{
P. L. Arun ${ }^{1}\left(\mathbb{D} \cdot\right.$ R Mathusoothana S Kumar $^{2}$
}

Received: 4 April 2020 /Revised: 21 September 2020 / Accepted: 7 October 2020/

Published online: 6 January 2021

(C) The Author(s) 2021

\begin{abstract}
Occlusion removal is a significant problem to be resolved in a remote traffic control system to enhance road safety. However, the conventional techniques do not recognize traffic signs well due to the vehicles are occluded. Besides occlusion removal was not performed in existing techniques with a less amount of time. In order to overcome such limitations, Non-linear Gaussian Bilateral Filtered Sorenson-Dice Exemplar Image Inpainting Based Bayes Conditional Probability (NGBFSEII-BCP) Method is proposed. Initially, a number of remote sensing images are taken as input from Highway Traffic Dataset. Then, the NGBFSEII-BCP method applies the Non-Linear Gaussian Bilateral Filtering (NGBF) algorithm for removing the noise pixels in input images. After preprocessing, the NGBFSEII-BCP method is used to remove the occlusion in the input images. Finally, NGBFSEII-BCP Method applies Bayes conditional probability to find operation status and thereby gets higher road safety using remote sensing images. The technique conducts the simulation evaluation using metrics such as peak signal to noise ratio, computational time, and detection accuracy. The simulation result illustrates that the NGBFSEII-BCP Method increases the detection accuracy by $20 \%$ and reduces the computation time by $32 \%$ as compared to state-of-the-art works.
\end{abstract}

Keywords Bayes conditional probability · Non-linear Gaussian bilateral filtering · Occlusion removal $\cdot$ Remote sensing images $\cdot$ Sorenson-Dice exemplar image inpainting $\cdot$ Traffic sign

P. L. Arun

aplpgme@gmail.com

R Mathusoothana S Kumar

rmsskdhujaa@gmail.com

1 Department of Electronics \& Communication Engineering, Research Scholar, Noorul Islam Centre for Higher Education, Kanyakumari, India

2 Department of Information Technology, Head of the Department, Noorul IslamCentre for Higher Education, Kanyakumari, India 


\section{Introduction}

Traffic control is a critical element to ensure efficiency and safety in a traffic system. It is affected by environmental conditions such as road signals, illumination, trees, weather conditions, and occlusion. Occlusion of traffic signs is one of the important tasks to reduce the risk of traffic accidents. One of the considerable applications of video-based supervision systems is traffic surveillance. Inpainting is a method to restore missing parts of the image using their background information. A lot of methods have been introduced for traffic image analysis and traffic flow control. But, the conventional techniques do not provide higher accuracy. Therefore, there is a requirement of a novel occlusion removal technique for efficient traffic control using remote sensing images. Hence, NGBFSEII-BCP Method is developed.

Traffic Sign Occlusion Detection (TSOD) method was presented in [8] to discover traffic signs using removing the occlusion objects in input images. A detection method was considered both reflectance and geometric features to capture traffic signs. A trajectory-based method was determined the driver's observation location and the corresponding observed traffic sign. A hidden point removal algorithm was developed to estimate the degree of occlusion. However, the amount of time required for efficient occlusion removal was higher. A fully convolutional network (FCN) was designed in [26] to carry out fast traffic sign detection. An efficient multi-class CNN model was trained for sign recognition by bootstrapping strategy. But, traffic sign detection accuracy was not adequate.

Image processing edge detection technique was introduced in [18] to address the traffic control problem. But, occlusion removal was not considered which impacts road safety. A novel method was presented in [9] to increase the efficiency of the smart traffic control system and controlling traffic in a single direction. But, the execution time was more.

A survey of various image processing techniques were designed in [6] for intelligent traffic management. In [13], codebook model and occlusion handling were presented for real-time traffic management. But, occlusion removal was not effectively carried out.

A color cubic feature called color cubic local binary pattern (CC-LBP) was presented in [12] to discover multiclass partial occluded traffic signs with high accuracy in real-time. However, the computation complexity was higher. A new approach was designed in [25] for system safety requirements. However, the safety measure rate was not at the required level.

An object-based MRF model with auxiliary label fields in [24] collects more macro and detailed data for enhancing the accuracy of segmentation. However, the execution time remained unaddressed. Static occlusion discovery and handling were performed in [16] with the help of transportation videos. However, the time complexity was very higher.

The proposed work is introduced with the new features of Sorenson-Dice Exemplar Image Inpainting (SEII) algorithm and Bayes conditional probability. SEII algorithm to eradicate the occlusion on the input image. Bayes conditional probability is applied to improve accuracy.

\subsection{Research gap}

The issues reviewed from the above existing traffic control works are computation time was not minimized during localization. The existing method underwent many difficulties to perform occlusion removal with higher accuracy. However, the time complexity was not 
minimized considerably. The designed scheme was not considered occlusion removal. But, the safety measure rate was not improved. In order to address these problems, NGBFSEII-BCP Method is introduced.

\subsection{Problem statement}

In a traffic control system, many conventional research works were developed for traffic image analysis and traffic flow control. But, the accuracy of detection using traditional methods is not sufficiently improved. Therefore, few research works have been implemented to perform preprocessing of images. However, the amount of time needed for efficient occlusion removal was not improved. Thus, it failed to increase the detection performance of traffic controls. The above issues are overcome by introducing the proposed Non-linear Gaussian Bilateral Filtered Sorenson-Dice Exemplar Image Inpainting Based Bayes Conditional Probability (NGBFSEIIBCP) method.

NGBFSEII-BCP method is introduced to enhance the road traffic control performance via an occlusion removal using remote sensing images. Initially, the NGBFSEII-BCP method takes a number of remote sensing images as input which is collected from a highway traffic dataset. Then, the NGBFSEII-BCP method uses the Non-linear Gaussian Bilateral Filtering (NGBF) algorithm to eradicate the noise pixels in input images. After preprocessing, the NGBFSEII-BCP method applies the Sørensen-Dice Exemplar Image Inpainting algorithm to eliminate the occlusion in the input images. Finally, NGBFSEII-BCP method utilizes Bayes conditional probability to discover operation status (i.e. traffic sign) and thereby efficiently controls the highway traffic on roads by using remote sensing images.

\subsection{Objective of the research works}

The main objective of the research work described as follows,

1) To enhance the road traffic control performance through an occlusion removal as compared to state-of-the-art works, the NGBFSEII-BCP method is proposed. The NGBFSEIIBCP method is introduced by integrating the NGBF, SEII, and BCP algorithms.

2) To remove the noisy pixels with higher peak signal to noise ratio as compared to existing works, Non-Linear Gaussian Bilateral Filtering (NGBF) algorithm is proposed in the NGBFSEII-BCP method,

3) To perform the occlusion removal process with minimum time as compared to state-ofthe-art works, Sorenson-Dice Exemplar Image Inpainting algorithm (SEII) is proposed in the NGBFSEII-BCP method.

4) To find operation status with improved detection accuracy as compared to state-of-the-art works, Bayes conditional probability (BCP) is proposed in the NGBFSEII-BCP method.

\subsection{Contribution of research work}

In order to enhance the road traffic control performance efficiently, proposed method is introduced. First, preprocessing is performed by using the NGBF algorithm. By using this algorithm, the noise pixel in the images is reduced. Next, Sorenson-Dice Exemplar Image Inpainting algorithm is utilized to eliminate the occlusion in the input images. Then, Bayes 
conditional probability is employed to find operation status and accurately detected as traffic signs.

The paper is organized as follows. Section 2, reviews the related works. Section 3, the detailed process of the NGBFSEII-BCP Method is explained using an architecture diagram. Section 4 describes the simulation settings. The comparative result analysis of the proposed NGBFSEII-BCP method is discussed in Section 5. Finally, the paper concluded in Section 6.

\section{Literature survey}

Motion Discovery and Running Gaussian Average (MDRGA) recursive technique was designed in [15] for high precision traffic monitoring alert system. But, the execution time was more. A graph-based tripartite (3D) Hungarian algorithm was employed in [21] to increase the performance of highway traffic video analytics. However, the false-positive rate of traffic signs detection was more.

A survey of different techniques designed for detection and handling of occlusion in images was analyzed in [3]. Traffic Sign Visual Recognizability Evaluation Model (TSVREM) was developed in [22] for enhancing traffic safety. But, the occlusion detection was not improved.

An Automatic Traffic Sign Detection was performed in [20] to increase the stability and reliability of transportation safety. This designed work consists of three working stages namely, image preprocessing, detection, and recognition. However, the processing time was not reduced. A novel method was designed in [19] for efficient detection and recognition of traffic signs from the image. The $2 \mathrm{D}$ and $3 \mathrm{D}$ techniques are used to improve the detection performance. But, the 3D localization of the signs was not considered.

To remove occluded objects and visualize hidden objects in the images an efficient PTSIOR mechanism is developed in [1]. It utilizes texture synthesis method. A super perception system of intelligent vehicles was developed in [11] for perceiving occluded street objects by gathering images from video streams. However, the accuracy of the projection model was not enhanced.

A novel method was presented in [2] for traffic signs discovery using google street view images for roadway inventory management. But, the processing time was more. A multilevel framework was implemented in [23] to remove vehicle occlusion with the application of a bidirectional occlusion reasoning algorithm. This framework includes three levels such as the intraframe, interframe and tracking levels. However, the computation cost was higher.

Unified PCM framework (UniPCM) was introduced in [7] for removing fast partially occluded objects in high-resolution remote sensing images with minimal time. But, time consumption was not reduced. A CNN-based forensic framework was introduced in [10] to discover image operations. But, the detection performance was not improved. An information theoretical framework was developed in [5] to quantify the detectability of one particular operation in multiple chains. However, the processing time was not reduced. A novel method was introduced in [17] to choose the optimal feature set and optimal classifier. But, the accuracy was not improved.In [4] objective evaluation framework was presented to attain 
privacy protection and utility by comparing with the standard computer vision tasks. However, computational time was higher.

The existing works does not use the dice similarity measure to calculate the similarity values. The proposed work uses dice similarity to compute the similarity value with minimum time. Various research works use Naive Bayes, the occlusion detection performance was not improved. Therefore, in proposed Bayes conditional probability, the accuracy is improved.

\section{Non-linear gaussian bilateral filtered Sorenson-dice exemplar image inpainting based Bayes conditional probability method}

Various research works focus on occlusion removal for traffic flow controls. Image inpainting technology is used to fill the missing region in the input image. But, the existing works fail in performing occlusion removal due to accuracy, time complexity, and false positive rate. In order to tackle these issues, NGBFSEII-BCP method is introduced. This method is introduced to improve the road traffic control performance through an occlusion removal using remote sensing images. On the contrary to state-ofthe-art works, this method is proposed by combining the Non-linear Gaussian Bilateral Filtering and Sorenson-Dice exemplar image inpainting and Bayes conditional probability. The architecture diagram of NGBFSEII-BCP is presented in Fig. 1.

NGBFSEII-BCP method attains higher road safety with minimal computational complexity. In Fig. 1, initially, this method takes a number of sensed images from a highway traffic dataset as input. After getting the input, it utilizes a non-linear Gaussian bilateral filtering algorithm. During the filtering process, it removes the noise pixels

Fig. 1 Architecture Diagram of NGBFSEII-BCP Method for Remote Traffic Control

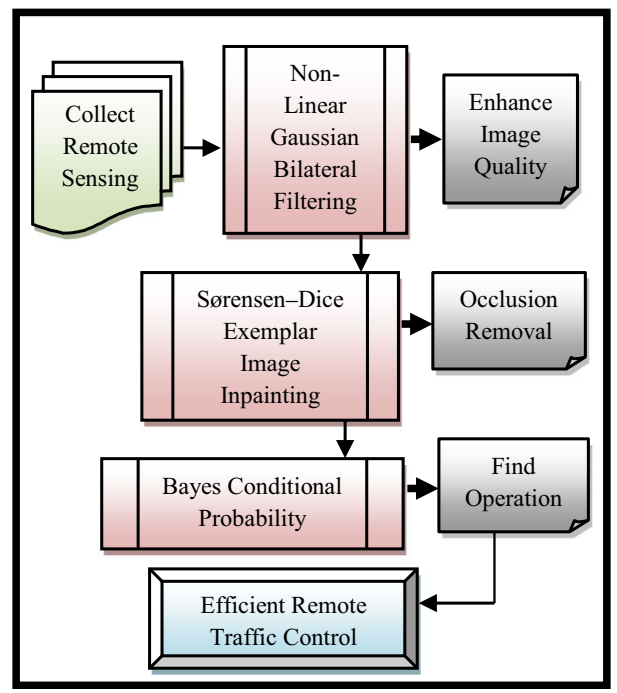


that exist in the input images and returns enhanced quality images for efficient occlusion removal.Then, the NGBFSEII-BCP method employs the Sorenson-Dice exemplar image inpainting algorithm that significantly removes the occlusion in input images. Finally, the proposed method used Bayes conditional probability [17] to identify operation status and thereby efficiently control the traffic occurrences on road as compared to state-of-the-art works. The detailed process of the NGBFSEII-BCP method is described in the below subsections.

\section{a. Non-linear Gaussian bilateral filter}

In NGBFSEII-BCP Method, NGBF algorithm is proposed to increase the quality of input remote sensing image. On the contrary to conventional works, the NGBF algorithm is designed with the application of Gaussian function in Bilateral Filtering. The process of the NGBF algorithm is depicted in Fig. 2.

The NGBF algorithm is used to carry out the preprocessing in the NGBFSEII-BCP method. As depicted in the Fig. 2, the NGBF algorithm initially obtains a number of remote sensing images as input and then computes weight based on spatial closeness and intensity differentiation. Consequently, the NGBF algorithm changes the noise pixels that are presented in input images with the weight value. From that, the NGBF algorithm efficiently eliminates the noise with a higher peak signal to noise ratio.

Let us consider the number of remote sensing images are denoted as ' $\alpha_{i}=\alpha_{1}, \alpha_{2}, \ldots, \alpha_{z}$ '. Here, ' $z$ ' represents the total number of images. Hence, non-linear Gaussian bilateral filtering is mathematically performed using the Eq. (1).

$$
\alpha^{\text {filtered }}(\beta)=\frac{1}{\mathrm{n}_{\mathrm{p}}} \sum_{\alpha_{\mathrm{i}}} \alpha\left(\beta_{\mathrm{i}}\right) \mathrm{a}\left(\left\|\alpha\left(\beta_{\mathrm{i}}\right)-\alpha(\beta)\right\|\right)
$$

In Eq. (1), ' $\alpha$ filtered' denotes the filtered image (i.e. higher quality remote sensing image) and ' $\alpha$ ' represents the input image. Here, ' $\beta$ ' points out the coordinates of the current pixel of input

Fig. 2 NGBF process for preprocessing

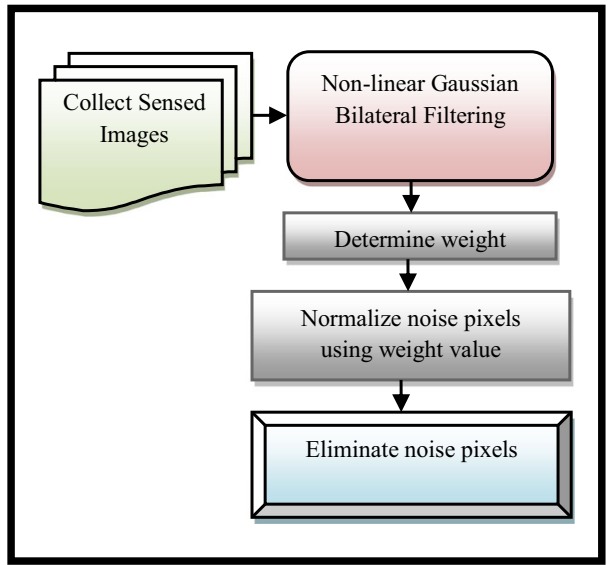


remote sensing image, ' $a$ ' is the range kernel for smoothing dissimilarities in intensities (i.e. Gaussian function). Here, ' $n_{p}$ ' represents the normalization terms which is calculated mathematically using Eq.(2)

$$
\mathrm{n}_{\mathrm{p}}=\sum_{\beta_{\mathrm{i}}} \mathrm{a}\left(\left\|\alpha\left(\beta_{\mathrm{i}}\right)-\alpha(\beta)\right\|\right) \mathrm{b}\left(\left\|\beta_{\mathrm{i}}-\beta\right\|\right)
$$

With the help of the Eq. (2), NGBF preserves the image energy. Here, 'b' indicates the spatial kernel for smoothing differences in coordinates (i.e. Gaussian function). Subsequently, NGBF computes a weight according to spatial closeness and intensity differentiation. Let us assume a noise pixel positioned at '(o,p)' that needs to be normalized in remote sensing image with its nearby pixels located at '(q,r)'. From that, weights ' $\tau(\mathrm{o}, \mathrm{p}, \mathrm{q}, \mathrm{r})$ ' is estimated using Eq.(3)

$$
\tau(\mathrm{o}, \mathrm{p}, \mathrm{q}, \mathrm{r})=\exp \left(-\frac{(\mathrm{o}-\mathrm{q})^{2}+(\mathrm{p}-\mathrm{r})^{2}}{2 \mathrm{x}_{\mathrm{d}}^{2}}-\frac{\|\alpha(\mathrm{o}, \mathrm{p})-\alpha(\mathrm{q}, \mathrm{r})\|^{2}}{2 \mathrm{y}_{\mathrm{r}}^{2}}\right)
$$

In Eq. (3), ' $\mathrm{x}_{\mathrm{d}} \mathrm{x}_{\mathrm{d}}, \mathrm{y}_{\mathrm{r}} \mathrm{y}_{\mathrm{r}}$ ' denotes smoothing parameters and ' $\alpha(\mathrm{o}, \mathrm{p})$ ' and ' $\alpha(\mathrm{q}, \mathrm{r})$ ' represents the intensity of pixels ' $(\mathrm{o}, \mathrm{p})$ ' and ' $(\mathrm{q}, \mathrm{r})$ ' respectively. After determining the weights, NGBF normalize the noise pixels of each input remote sensing image with the help of the Eq. (4)

$$
\alpha_{\mathrm{D}}(\mathrm{o}, \mathrm{p})=\frac{\sum_{\omega, \varphi} \alpha(\mathrm{q}, \mathrm{r}) \tau(\mathrm{o}, \mathrm{p}, \mathrm{q}, \mathrm{r})}{\sum_{\mathrm{q}, \mathrm{r}} \tau(\mathrm{o}, \mathrm{p}, \mathrm{q}, \mathrm{r})}
$$

From Eq. (4), ' $\alpha_{D}$ ' denotes the de-noised intensity of pixel ' $(o, p)$ ' in input remote sensing image. With the support of the above process of the NGBF algorithm, NGBFSEII-BCP method enhances the quality of each input remote sensing image through removing the noisy pixel with higher peak signal to noise ratio with minimal time.

\section{b. Sorenson-dice exemplar image inpainting}

In the NGBFSEII-BCP method, SEII algorithm is designed for removing the occlusion in an input remote sensing image. On the contrary to conventional works, the SEII algorithm is proposed by combining the Sorenson-Dice similarity coefficient in exemplar based image inpainting technique. In the proposed SEII algorithm, Sorenson-Dice similarity coefficient metric is very essential to identify the most similar patch from the source region. Therefore, the Sorenson-Dice similarity coefficient is employed in the SEII algorithm. With the help of the Sorenson-Dice similarity coefficient metric, the SEII algorithm enhances the similarity between patches and computes the similarity between the target patch and each source patch. From that, the SEII algorithm effectively takes away the occlusions in input remote sensing images with a minimal amount of time complexity. The process of the SEII algorithm is demonstrated in Fig. 3. 
Fig. 3 SEII process for Occlusion Removal

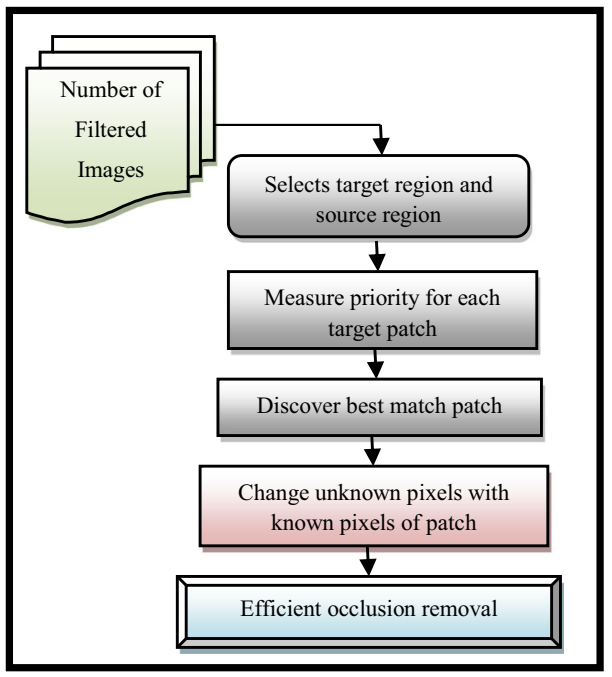

The SEII algorithm is used to perform the occlusion removal process. In Fig. 3, the SEII algorithm at first gets the number of filtered remote sensing images as input. Then, the SEII algorithm finds the target region and source region for each input image. Next, the algorithm determines the priority for each target patch and thereby finds out the best match patch by considering pixels similarities. Finally, the SEII algorithm changes unknown pixels with known pixels of the patch. As a result, the SEII algorithm precisely removes the occlusions in input remote sensing images.

Let us consider an input remote sensing image represented by ' $\alpha$ ' and the target region is denoted by ' $\beta$ ' and its contour is indicated as ' $\partial \beta$ '. The source region, the target patch is denoted by ' $\gamma_{\mathrm{p}}$ ' and the source path is represented by ' $\delta_{\mathrm{p}}$ '. In the proposed NGBFSEII-BCP method, the default mask size of ' $9 * 9$ ' pixels is considered as a patch region. The format of ' 9 x9' default mask size is show in the Fig. 4.

In Fig. 4, each box represents various pixel values presented in the images. Initially, the SEII algorithm chooses the target region ' $\beta$ ' of an image and the residual part of the image is

Fig. $49 \times 9$ window

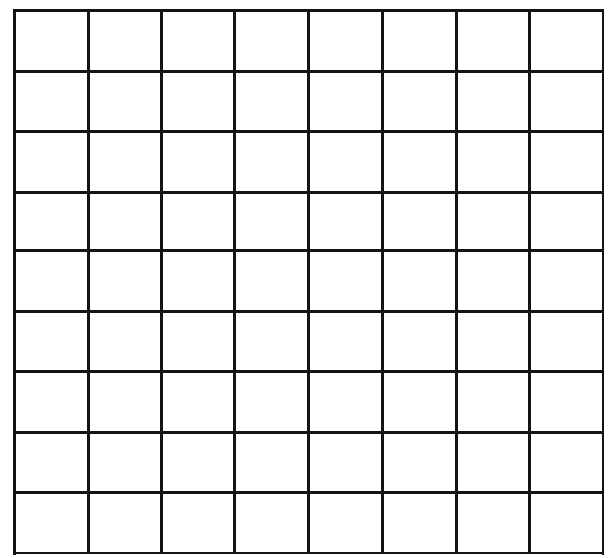


considered as the source region ' $\delta$ '. Next, the SEII algorithm finds the contour ' $\partial \beta$ ' of the target region ' $\beta$ '. For each target path ' $\gamma_{p}$ ' centered at the point ' $\rho(p \in \partial \beta)$ ', then the SEII algorithm determines priority ' $\rho(\mathrm{p})$ ' using the Eq. (5),

$$
\rho(\mathrm{p})=\mu(\mathrm{p}) \times \omega(\mathrm{p})
$$

In the above Eq. (5), ' $\mu(\mathrm{p})$ ' estimate the amount of reliable information in the patch ' $\gamma_{\mathrm{p}}$ ' and ' $\mu(\mathrm{p})$ ' is given by the Eq. (6).

$$
\mu(p)=\frac{\sum_{q \in \gamma_{p} \cap \varnothing \mu}(q)}{\left|\gamma_{p}\right|}
$$

From Eq. (6), ' $\left|\gamma_{\mathrm{p}}\right|$ ' points out the number of pixels in the patch ' $\gamma_{\mathrm{p}}$ '. In the SEII algorithm, ' $\mu(p)=1$ ' if ' $p \in \Phi$ ' and ' $\mu(p)=0$ 'if ' $p \in \beta$ '. Consequently, the data term ' $\omega(p)$ ' in the SEII algorithm represents a strength function of isophotes hitting the contour ' $\partial \beta$ ' each iteration which is mathematically defined by Eq. (7).

$$
\omega(p)=\frac{\left|\nabla \alpha_{\mathrm{p}}^{\perp} \cdot \overrightarrow{\mathrm{z}}_{\mathrm{p}}\right|}{c}
$$

In the Eq. (7), 'c' indicates the normalization factor and has a value 255 . Here, ' $\vec{z}_{\mathrm{p}}$ ' represents the unit normal vector that is orthogonal to current contour ' $\partial \beta$ ' at ' $p$ ' and ' $\perp$ ' refers orthogonal operator. After determining the patch ' $\gamma_{\mathrm{p}}$ ' with the highest priority, SEII algorithm finds a best match ' $\gamma_{\mathrm{q}}$ ' from the source region using Eq. (8).

$$
\gamma_{q}=\operatorname{argmax} \$\left(\gamma_{p}, \gamma_{q}\right)
$$

In Eq. (8), ' $\$\left(\gamma_{\mathrm{p}}, \gamma_{\mathrm{q}}\right)$ ' denotes the Sorenson-Dice similarity. On the contrary to existing algorithm, the proposed NGBFSEII-BCP method uses Sorenson-Dice similarity for measuring the pixels similarities between the two patchs ' $\gamma_{\mathrm{p}}$ ' and ' $\gamma_{\mathrm{q}}$ ' to find the occlusion. A mathematical equation of the Sorenson-Dice similarity is given by Eq. (9).

$$
\$\left(\gamma_{p}, \gamma_{q}\right)=\frac{\gamma_{p} \cap \gamma_{q}}{\left|\gamma_{p}\right| *\left|\gamma_{q}\right|}
$$

In the Eq. (9), ' $\$\left(\gamma_{\mathrm{p}}, \gamma_{\mathrm{q}}\right)$ ' is the Sorenson-Dice similarity, intersection symbol ' $\cap$ ' signifies a mutual dependence between pixels in patch ' $\gamma_{\mathrm{p}}$ ' and ' $\gamma_{\mathrm{q}}$ ' whereas ' $\left|\gamma_{\mathrm{p}}\right|$ 'and ' $\left|\gamma_{\mathrm{q}}\right|$ ' indicates the cardinalities between the two-pixel features. The Sorenson-Dice similarity values ' $\$\left(\gamma_{\mathrm{p}}, \gamma_{\mathrm{q}}\right)^{\text {' is }}$ in a range between ' 0 ' and ' 1 '. If the pixels in two patch ' $\gamma_{\mathrm{p}}$ ' and ' $\gamma_{\mathrm{q}}$ ' are similar, then the output value is ' 1 '. When the two pixels in two patch ' $\gamma_{\mathrm{p}}$ ' and ' $\gamma_{\mathrm{q}}$ ' are dissimilar, the output value is ' 0 '. The dissimilar pixels in the patches are called as occlusion and it is removed from the image. 
After finding the best match patch, the SEII algorithm changes the unknown pixels of patch ' $\gamma_{\mathrm{p}}$ ' with the help of known pixels of patch ' $\gamma_{\mathrm{q}}$ ' and subsequently updates confidence value using the Eq. (10).

$$
\mu(q)=\mu(p), \forall q \in \gamma_{p} \cap \beta
$$

The above processes of the SEII algorithm are continued until all the missing pixels in the input image are filled. Thus, the NGBFSEII-BCP method accurately removes the occlusions in input remote sensing images with a minimal amount of time.

\section{c. Bayes conditional probability}

After removing the occlusions, Bayes Conditional Probability (BCP) algorithm [17] is employed in the NGBFSEII-BCP method to determine operation status (i.e. traffic sign) and thereby efficiently controls the traffic on roads using remote sensing images. The BCP algorithm utilizes Bayes' theorem that identifies the probability of an event for each input remote sensing image by considering the prior knowledge of conditions that are associated with the event. The measured probability result helps for the BCP algorithm to analyze the status of the event. The probability result ' 1 ' represents that traffic sign is detected in the input remote sensing image whereas ' 0 ' denotes there is no traffic sign in the input remote sensing image. With assists of the discovered status of the event, finally, the BCP algorithm significantly performs the road traffic control process. Thus, the $\mathrm{BCP}$ algorithm increases the performance of remote traffic control as compared to conventional works. The process of the BCP algorithm is depicted in Fig. 5.

The BCP algorithm effectively performs a remote traffic control process. The BCP algorithm computes BCP for each input quality enhanced remote sensing images ' $\alpha_{i}$ ' as per the Eq.(11).

$$
\operatorname{Pr}(E / F)=\frac{P(F / E) P(E)}{P(F)}
$$

In Eq. (11), ' $\mathrm{E}$ ' and ' $\mathrm{F}$ ' represent the two events where ' $\mathrm{P}(\mathrm{E})$ ' is a probability of (E) is first event, the traffic sign is detected.P(F) is a the probability of $(\mathrm{F})$ is a second event traffic sign is not detected. Here, ' $P(E / F)$ ' indicates the probability of ' $E$ ' for given ' $\mathrm{F}$ '. If the result of ' $\operatorname{Pr}(\mathrm{E} / \mathrm{F})=1$ ', then the $\mathrm{BCP}$ algorithm considered that traffic sign is detected. Otherwise, the $\mathrm{BCP}$ algorithm considered that there is no traffic sign is found. Based on discovered operation status (i.e. traffic sign or not), the NGBFSEII-BCP method increases the traffic control performance as compared to existing works using remote sensing images.

The algorithm of the NGBFSEII-BCP Method.

Algorithm 1. Non-linear Gaussian Bilateral Filtered Sorenson-Dice Exemplar Image Inpainting Based Bayes Conditional Probability 
// Non-linear Gaussian bilateral filtering Sorenson-Dice exemplar image inpainting based Bayes conditional probability algorithm

Input: Number of remote sensing images ' $\alpha_{i}=\alpha_{1}, \alpha_{2}, \ldots, \alpha_{z}$ '

Output: Improved road traffic control with occlusion removal

\section{Step 1: Begin}

// Preprocessing

Step 2: for each sensed images ' $\alpha_{i}$ '

Step 3: $\quad$ Apply Non-Linear Gaussian Bilateral Filter using Eq. (1)

Step 4: $\quad$ Measure weight ' $\tau(o, p, q, r)$ ' using Eq. (3)

Step 5: $\quad$ Normalize the noise pixels with weight value using Eq. (4)

Step 6: $\quad$ Remove noise pixels

Step 7: End for

\section{// Occlusion Removal}

Step 8: $\quad$ for Each Filtered Image

Step 9: $\quad$ Selects target region as ' $\beta$ ' and source region as ' $\delta$

Step 10: while (all the missing pixels in the image are filled) do

Step 11: $\quad$ for each target patch ' $\gamma_{p}$ '

Step 12: $\quad$ Determines priority ' $\rho(p)$ ' using Eq. (5)

Step 13: $\quad$ Find best match patch ' $\gamma_{q}$ ' by considering pixels similarities using Eq.(8)

Step 14: $\quad$ Changes unknown pixels of patch ' $\gamma_{p}$ ' with known pixels of patch ' $\gamma_{q}$ '

Step 15: $\quad$ Remove occlusion in image

Step 16: $\quad$ End for

Step 17: $\quad$ End while

Step 18: End for

// Remote Traffic Control

Step 19: for each high-quality image

Step 20: $\quad$ Apply Bayes conditional probability using Eq. (11)

Step 21: $\quad$ if $(\operatorname{Pr}==1)$, then

Step 22: $\quad$ Traffic sign is detected

Step 23: $\quad$ else

Step 24: $\quad$ No Traffic sign

Step 25: $\quad$ End if

Step 26: End for

Step 27:End 
Algorithm 1 given above explains the step by step process of the NGBFSEII-BCP Method to find the traffic sign with a minimal amount of time utilization. This supports for NGBFSEII$\mathrm{BCP}$ method to effectively manage traffic on road using remote sensing images as compared to state-of-the-artwork.

\section{Simulation review}

In order to measure the performance of the proposed NGBFSEII-BCP method, the existing Traffic Sign Occlusion Detection (TSOD) method [8] and Fully Convolutional Network (FCN) [26] is implemented in MATLAB 2019A simulator using Highway Traffic Dataset [14]. This dataset contains a set of highway traffic videos collected remotely. The video is divided into a number of frames that are considered as remote sensing images to perform simulation work. In order to conduct the simulation purpose, this method takes a different number of remote sensing images in the range of 25-250.

\subsection{Simulation settings}

All the experiments are conducted with windows 10 Operating system 64 bit, processor intel core, RAM 4GB. The efficiency of the NGBFSEII-BCP Method is determined in terms of peak signal to noise ratio, computational time and detection accuracy. The simulation result of the NGBFSEII-BCP method is compared against two conventional methods namely Traffic Sign Occlusion Detection (TSOD) method [8] and Fully Convolutional Network (FCN) [26]. The proposed NGBFSEII-BCP method is used by highway traffic dataset [7].

\section{Result and discussions}

Fig. 5 BCP Algorithmic Process for Remote Traffic Control

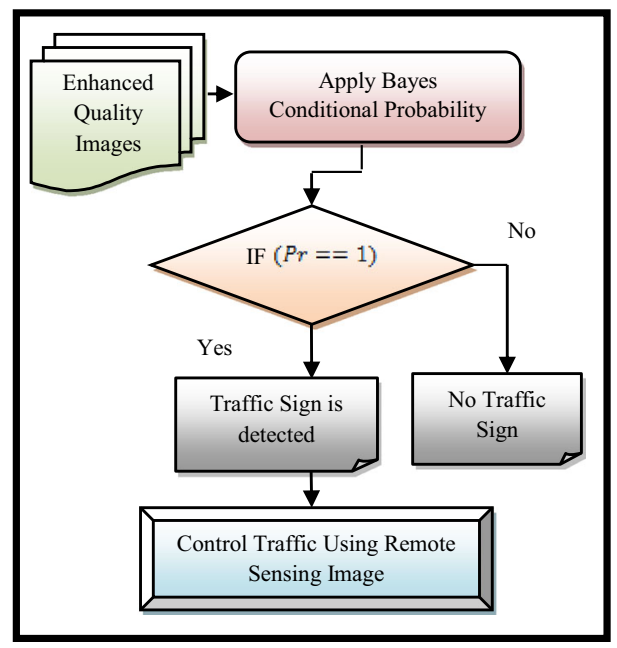


In this section, the simulation result of the proposed method is discussed. The efficacy of the method is compared with the existing Traffic Sign Occlusion Detection (TSOD) method [8] and fully convolutional network (FCN) [26] with help of parameters such as peak signal to noise ratio, computational time, and detection accuracy.

a. Peak signal to noise ratio

In the NGBFSEII-BCP method, Peak Signal to Noise Ratio (PSNR) computes the distinction between input noisy image and quality enhanced image after completing the preprocessing task. The PSNR is mathematically calculated as follows,

$$
\begin{aligned}
& P S N R=10 \log _{10} \frac{R^{2}}{M S E} \\
& M S E=\sum_{i=1}^{z}\left(\alpha_{i}-\alpha_{i}^{\prime}\right)
\end{aligned}
$$

From the above mathematical Eqs. (12) and (13), the mean square error 'MSE' is measured based on the cumulative squared error between the actual image size ' $\alpha$ ' and preprocessed image size ' $\alpha$ '. With the help of estimated mean square error using Eq. (13), then peak signalto-noise ratio 'PSNR' is determined using the maximum possible pixel value of the images $(R R=255)$. The Peak Signal to Noise Ratio (PSNR) is calculated in terms of decibel (dB).

The experimental result of the peak signal to noise ratio is depicted in Table 1.

A higher peak signal to noise ratio is achieved with the aid of the NGBF algorithm, calculate the weight depended on spatial closeness and intensity differentiation in the proposed NGBFSEII-BCP method. Then, the NGBF algorithm changes the noise pixels in input images with the weight value. Thus, the NGBF algorithm accurately eliminates the noise with a higher peak signal to noise ratio. This supports for proposed method to decrease the difference between input noisy image and quality enhanced image as compared to state-of-the-art works. As a result, PSNR is improved in the NGBFSEII-BCP method by $4 \%$ and $10 \%$ as compared to TSOD [8] and FCN [26].

\begin{tabular}{|c|c|c|c|}
\hline \multirow[t]{2}{*}{ Image Size (KB) } & \multicolumn{3}{|c|}{ Peak Signal to Noise Ratio (dB) } \\
\hline & NGBFSEII-BCP & TSOD & $\mathrm{FCN}$ \\
\hline 11.4 & 50.07 & 47.3 & 44.05 \\
\hline 12.1 & 47.307 & 45.21 & 42.56 \\
\hline 11.9 & 46.55 & 44.61 & 42.11 \\
\hline 12.2 & 45.21 & 43.52 & 41.67 \\
\hline 11.8 & 45.85 & 44.05 & 41.67 \\
\hline 12.0 & 43.52 & 42.11 & 40.53 \\
\hline 14.9 & 44.05 & 42.56 & 40.9 \\
\hline 13.8 & 46.55 & 44.61 & 42.56 \\
\hline 12.7 & 45.21 & 43.52 & 41.28 \\
\hline $\mathrm{s}$ & 43.03 & 41.69 & 39.83 \\
\hline
\end{tabular}

Table 1 Tabulation for Peak Signal to Noise Ratio 
b. Computational time

In NGBFSEII-BCP Method, Computational Time (CT) measures the amount of time required for removing the occlusions in remote sensing images. The computational time is mathematically obtained by Eq. (14),

$$
C T=z * T(R O S I)
$$

The computational time is determined in terms of milliseconds (ms). Here, ' $\mathrm{z}$ ' represents the total number of remote sensing images whereas 'T(ROSI)' indicates time utilized to remove the occlusions in a single image.

Figure 6 presents the impact of computational time with respect to a diverse number of remote sensing images in the range of 25-250 using three methods namely the NGBFSEIIBCP method, conventional TSOD [8], and FCN [26]. The proposed method achieves minimal computational time. This is owing to the application of the NGBF algorithm and SEII algorithm in the proposed NGBFSEII-BCP method on the contrary to conventional works.

With the application of the NGBF algorithm, the proposed NGBFSEII-BCP method improves preprocessing performance as it significantly removes the noise pixels. Besides the aid of the SEII algorithm, the proposed NGBFSEII-BCP method takes away the occlusions in input images with lesser time consumption. Hence, the proposed NGBFSEII-BCP method minimizes the computation time of occlusion removal by $27 \%$ and $36 \%$ as compared to TSOD [8] and FCN [26].

\section{c. Detection accuracy}

In NGBFSEII-BCP Method, Detection Accuracy '(DA)' determines the ratio of the number of remote sensing images that are accurately detected as a traffic sign or not to the total number of remote sensing images taken as input. The detection accuracy is evaluated by Eq. (15).

$$
D A=\frac{z_{C D}}{z} * 100
$$

From the Eq. (15), the detection accuracy of traffic sign using the proposed and existing two methods are estimated with respect to the different number of input images. The detection accuracy is computed in terms of percentage (\%). Here, ' $z$ ' denotes the total number of remote sensing images considered for experimental evaluation whereas ' $\mathrm{z}_{\mathrm{CD}}$ ' point out the number of remote sensing images that are correctly detected. The performance result analysis of traffic sign detection accuracy is depicted in Table 2.

Fig. 6 Performance Measure of Computational Time versus Number of Remote Sensing Images

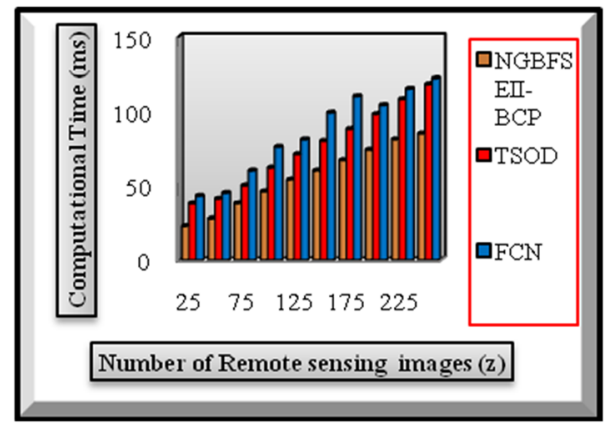


Table 2 Tabulation for Detection Accuracy

\begin{tabular}{llll}
\hline Number of Remote sensing images $(\mathrm{z})$ & \multicolumn{2}{l}{ Detection Accuracy $(\%)$} & \\
\cline { 2 - 4 } & NGBFSEII-BCP & TSOD & FCN \\
\hline 25 & 92 & 64 & 52 \\
50 & 90 & 74 & 70 \\
75 & 95 & 85 & 81 \\
100 & 93 & 85 & 78 \\
125 & 96 & 88 & 82 \\
150 & 94 & 88 & 79 \\
175 & 95 & 87 & 80 \\
200 & 91 & 85 & 79 \\
225 & 93 & 84 & 78 \\
250 & 96 & 89 & 84 \\
\hline
\end{tabular}

By using the concepts of NGBF, SEII, BCP algorithms, the proposed NGBFSEII-BCP method exactly find operation status (i.e. traffic sign) by measuring the probability of an event based on prior knowledge of conditions that related to the event for each input remote sensing image. Therefore, the proposed NGBFSEII-BCP method enhances detection accuracy by $14 \%$ and $25 \%$ as compared to TSOD [8] and FCN [26].

\section{Conclusion}

An effective NGBFSEII-BCP method is proposed to improve the performance of road traffic control via occlusion removal. The goal of the NGBFSEII-BCP method is achieved with the application of NGBF, SEII and BCP. The proposed NGBFSEII-BCP method precisely removes noisy pixels in input remote sensing images with enhanced peak signal to noise ratio with the application of the NGBF algorithm. Further, the proposed method decreases the amount of time needed for efficient occlusion removal with the help of the SEII algorithm. Also, the proposed method improves the ratio of a number of remote sensing images that are accurately detected as traffic signs by using the BCP algorithm. The simulation of NGBFSEIIBCP Method is estimated in terms of peak signal to noise ratio, computational time, and detection accuracy and compared with respect to a different number of remote sensing images. The simulation result demonstrates that the proposed method provides better performance with an enhancement of detection accuracy and minimization of computational time for remote traffic control. In future, the work of our proposed work is also proceed using wavelet preprocessing to extract edge direction information and remove the occlusion for detecting the high traffic sign. Robust correlation-based method is used to detect the traffic sign for improving the accuracy.

Open Access This article is licensed under a Creative Commons Attribution 4.0 International License, which permits use, sharing, adaptation, distribution and reproduction in any medium or format, as long as you give appropriate credit to the original author(s) and the source, provide a link to the Creative Commons licence, and indicate if changes were made. The images or other third party material in this article are included in the article's Creative Commons licence, unless indicated otherwise in a credit line to the material. If material is not included in the article's Creative Commons licence and your intended use is not permitted by statutory regulation or exceeds the permitted use, you will need to obtain permission directly from the copyright holder. To view a copy of this licence, visit http://creativecommons.org/licenses/by/4.0/. 


\section{References}

1. Arun PL, Mathusoothana SK (2019) Prewitt texture filter synthesis for efficient image occlusion removal to visualize hidden objects. J Adv Res Dyn Control Syst 11:1052-1061

2. Balali V, Rad AA, Golparvar-Fard M(2015) Detection, classification, and mapping of U.S. traffic signs using google street view images for roadway inventory management, Vis Eng, Springer, 3(15):1-18

3. Chandel H, Chandel H (2015) Occlusion detection and handling: a review. Int J Comput Appl (0975-8887), 120(10):33-38

4. Erdelyi A, Winkler T, Rinner B (2018) privacy Protection vs. utility in visual data an objective evaluation framework. Multimed Tools Appl 78:2285-2312

5. Gao S, Liao X, Liu X (2019) Real-time detecting one specific tampering operation in multiple operator chains. J Real-Time Image Proc 16:741-750

6. Gulati I, Srinivasan R (2019) Image processing in intelligent traffic management. Int J Recent Techno Eng (IJRTE) 8(2S4):213-218

7. Highway Traffic Dataset (n.d.) : http://sbmi2015.na.icar.cnr.it/SBIdataset.html

8. Huang P, Cheng M, Chen Y, Luo H, Wang C, Li J (2017) Traffic sign occlusion detection using mobile laser scanning point clouds. IEEE Trans Intell Transp Syst 18(9):2364-2376

9. Jadhav P, Kelkar P, Patil K, Thorat S (2016) Smart traffic control system using image processing. Int Res J Eng Technol (IRJET) 03(3):1207-1211

10. Liao X, Li K, Zhu X, Liu KJR (2020) Robust detection of image operator chain with two-stream convolutional neural network. IEEE J Sel Top Sign Proces 14(5):955-968

11. Liu C, Chang F, Liu C (2016) Occlusion-robust traffic sign detection via cascaded colour cubic feature. IET Intell Transp Syst 10(5):354-360

12. Liu W, Wei L, and Li Y(2018) Occluded street objects perception algorithm of intelligent vehicles based on the 3D projection model. Hindawi, J Adv Transp , Article ID 1547276: 1-11, 2018

13. Moutakki Z, Ouloul IM, Afdel K, Amghar A (2017) Real-time video surveillance system for traffic management with background subtraction using codebook model and occlusion handling. Trans Telecommun 18(4):297-306

14. Qiu S, Wen G, Liu J, Deng Z, Fan Y (2018) Unified partial configuration model framework for fast partially ocluded object detection in high-resolution remote sensing images. Remote Sens 10(3):1-23

15. Reena S, Ranjani JJ (2016) High precision traffic monitoring alert system using image background subtraction. Indian J Sci Technol 9(48):1-6

16. Shreve M, Bernal E A, Li Q, Loce R P(2015) Static Occlusion Detection and Handling in Transportation Videos. 2015 IEEE 18th International Conference on Intelligent Transportation Systems:638-643

17. Soni R, Kumar B, Chand S (2019) Optimal feature and classifier selection for text region classification in natural scene images using Weka tool. Multimed Tools Appl 78:31757-31791

18. Srinivas P, Malathilatha YL, Prasad MVNK (2013) Image processing edge detection technique used for traffic control problem. Int J Comput Sci Inf Technol 4(1):17-20

19. Timofte R, Zimmermann K, Gool LV(2014) Multi-view traffic sign detection, recognition, and 3D localization.Machine Vis Appl Springer, 25(3):633-647

20. Wali SB, Hannan MA, Hussain A, Samad SA(2015) An automatic traffic sign detection and recognition system based on colour segmentation, shape matching, and SVM. Mathematical problems in engineering, Hindawi publishing corporation, Article ID 250461:1-11, 2015

21. Wan Y, Huang Y, Buckles B(2014) Camera calibration and vehicle tracking: highway traffic video analytics. Trans Res Part C: Emerg Technol, Elsevier, 44: 202-213

22. Zhang S, Wang C, Lin L, Wen C, Yang C, Zhang Z, Li J (2019) Automated visual recognizability evaluation of traffic sign based on 3D LiDAR point clouds. Remote Sens 11(12):1185-1190

23. Zhang W, Wu QMJ, Yang X, Fang X (2008) Multilevel framework to detect and handle vehicle occlusion. IEEE Trans Intell Transp Syst 9(1):161-174

24. Zheng C, Zhang Y, Wang L (2017) Semantic segmentation of remote sensing imagery using an object-based Markov random field model with auxiliary label fields. IEEE Trans Geosci Remote Sens 55(5):3015-3028

25. Zhou L, Cao G, Li Y, Shang Y (2016) Change detection based on conditional random field with region connection constraints in high-resolution remote sensing images. IEEE J Select Topi Appl Earth Observations Remote Sens 9(8):3478-3488

26. Zhu Y, Zhang C, Zhou D, Wang X, Bai X, Liu W, (2016) Traffic sign detection and recognition using fully convolutional network guided proposals. Neurocomputing, Elsevier, 214:758-766

Publisher's note Springer Nature remains neutral with regard to jurisdictional claims in published maps and institutional affiliations. 